\title{
INFLUENCIA DEL ENTRENAMIENTO CON PESAS SOBRE LA MEMORIA Y EL TIEMPO DE REACCIÓN
}

\author{
Magally Márquez Barquero y María Clara Rodríguez Salazar \\ Escuela de Educación Física y Deportes \\ Universidad de Costa Rica, San José, Costa Rica \\ E-mail:maga13@costarricense.cr
}

\begin{abstract}
Resumen
Márquez Barquero, M. y Rodríguez Salazar, M. C. (2002). Influencia del entrenamiento con pesas sobre la memoria y el tiempo de reacción. Revista de Ciencias del Ejercicio y la Salud, 2(1), 31-40. El objetivo de la presente investigación fue determinar si una sesión de ejercicio con pesas, a diferentes cargas, afecta la memoria y el tiempo de reacción en un grupo de mujeres mayores de 50 años. Se trabajó con 20 sujetos, divididas en dos grupos: entrenadas y sedentarias. Cada sujeto realizó una sesión para cada una de las tres condiciones experimentales: $80 \%$ de fuerza máxima, 50\%, y control. En cada una de estas se aplicó el Verbal Scrip Digit Span y el test de la mano de Nelson, antes y después del trabajo con pesas. No se hallaron diferencias significativas entre los grupos, aunque sí las hubo entre las mediciones de pre-test y post-test, incluso en el grupo control por lo que se infiere que los cambios obtenidos fueron debidos a aprendizaje o a otro factor y no al ejercicio. PALABRAS CLAVES: ejercicio físico, entrenamiento con pesas, memoria, tiempo de reacción, adulto mayor.
\end{abstract}

\section{INTRODUCCIÓN}

La actividad física está asociada con estados de salud física, psicológica y social; se le considera un promotor de salud en general en tanto ha demostrado ser efectiva para la prevención y el tratamiento de variadas enfermedades. Tanto la práctica deportiva como los programas de ejercicios son las principales posibilidades de intervención que mayor atención han recibo por parte de los científicos (Lox, Burns, Treasure y Wasley, 1999; Clark, 1996; Maxwell, 1994; Huges, 1973; Valliant y Asu, 1984; Alfaro y Salazar, 2001).

Existe suficiente evidencia respecto a los beneficios del ejercicio sobre diferentes variables como la fuerza, flexibilidad, composición corporal y resistencia cardiovascular (Morel, 1996; Meléndez, 2000; Lox y cols. 1999; Márquez y Campos, 2001), cualidades importantes tanto para el desarrollo infantil y juvenil como para el bienestar del adulto y adulto mayor (Sallis, 2000; Meléndez, 2000).
La relación entre la actividad física y los procesos cognitivos se ha constituido en una importante área de investigación durante las últimas dos décadas; los resultados reafirman la importancia de la práctica del ejercicio como un medio para mejorar la calidad de vida en este aspecto (García, Navarro y Ruiz, 1996, Sallis, 2000, Pollock y Evans, 1998). Estudios en los que se ha utilizado el ejercicio como tratamiento, sugieren que existe una estrecha relación entre el nivel de aptitud física y el funcionamiento cognitivo (Aguirre, 1999; Morel, 1996). Perrig-Chiello, Perrig, Ehrsam, Staehlin y Krings (1998), sostienen igualmente que el nivel cognitivo está asociado positivamente con el nivel de actividad física.

Se ha postulado como hipótesis que el ejercicio mejora la capacidad mental causando un efecto favorable en el metabolismo de los neurotransmisores acetilcolina, dopamina, norepinefrina y serotonina. Otra hipótesis que intenta explicar el mecanismo mediante el cual el 
ejercicio favorece la actividad cognitiva, plantea que éste incrementa el flujo sanguíneo hacia el cerebro aumentando a su vez la distribución de nutrientes como la glucosa y el oxígeno (Emery y Blumenthal, 1991; Aguirre, 1999, Etnier, Salazar, Landers, Petruzzello, Han y Novel, 1997).

Los cambios morfológicos 0 funcionales que presenta el organismo como consecuencia del ejercicio, se pueden catalogar como efectos crónicos o agudos. Al someter a un organismo a un proceso contínuo de adaptación, se produce un efecto crónico; se trata de cambios a largo plazo, alteraciones progresivas que permanecen después de cesar el ejercicio y que implican una adaptación relativamente estable del organismo. Por el contrario, el efecto agudo es la respuesta inmediata a un estímulo, que desaparece tan pronto desaparece el estímulo que la provocó, es la respuesta temporal a una sesión única, no a un proceso (García, Navarro y Ruiz, 1996).

Al discutir el efecto agudo del ejercicio sobre la función cerebral, se sugiere que el trabajo submáximo puede mejorar la función cognitiva y la viveza mental (Bly y Rumelhart, 1999). Etnier y cols. (1997) concluyen que cuando se analiza tal efecto, los resultados pueden ser afectados tanto por la complejidad de la tarea cognitiva como por la intensidad del ejercicio.

Moreira (1993), al estudiar los efectos del ejercicio sobre variables cognitivas, afirma que el efecto agudo del ejercicio sobre los procesos cognitivos es mayor que el efecto crónico, ya que hay un incremento de la función cognitiva después de una sesión de ejercicio físico. Por su parte, Sanabria (1995), mediante un metaanálisis sobre los efectos de la actividad física sobre la función mental, determinó que el ejercicio aeróbico, tanto agudo como crónico, produce un efecto significativo sobre diferentes procesos cognitivos (tiempo de reacción, memoria, inteligencia y creatividad).

Aguirre (1999) realizó un estudio con 28 adultos mayores con quienes desarrolló un programa de entrenamiento con pesas que incluyó press de banca horizontal y extensión de rodillas. Dentro de los hallazgos de su estudio se comprobó que el entrenamiento con pesas a intensidades del $30 \%$ de 5 RM produce beneficios en la memoria auditiva, la discriminación perceptual, el tiempo de reacción y el estado mental general en el adulto mayor mientras se mantenga físicamente activo, pero estos beneficios tienden a desaparecer una semana después de cesar el ejercicio. Por lo tanto, el entrenamiento con pesas es una actividad física útil para atenuar el proceso de deterioro causado por la edad, tanto a nivel físico como mental.

Para Puente, Moya y Mayor (1998), las funciones cognitivas comprenden los procesos de percepción, atención, memoria, aprendizaje, pensamiento y lenguaje. El tiempo de reacción es básicamente un proceso perceptivo de nivel inferior de procesamiento de información, mientras que la memoria requiere niveles superiores que determinarán los diferentes tipos de memoria.

El tiempo de reacción (TR) se define como el tiempo transcurrido entre inicio de un estímulo e inicio de la respuesta solicitada a un sujeto. En muchas situaciones corrientes de la vida diaria, la velocidad de reacción es de vital importancia, las demandas del medio le exigen a la persona la adecuación temporal de la respuesta que se emite. Para su medición se han empleado diversidad de aparatos y equipos que no difieren en su esquema básico: la presentación del estímulo elicitador de la respuesta activa un cronómetro que se detiene en el instante en que el sujeto presenta la respuesta demandada. Su medida se ha tomado básicamente en dos modalidades: manual, cuya respuesta se considera simple y pedal considerada compleja. Depende de la excitación del receptor sensorial involucrado en la percepción de la señal, así como del tiempo de transmisión de la señal del receptor al sistema nervioso central y de éste a la 
traducción en ejecución motriz. Factores de índole sensorial y perceptiva pueden alterar dramáticamente el TR (Roca, 1997).

El tiempo de reacción ha sido una de las variables más estudiadas desde el campo de la psicología del deporte (Riera y Cruz, 1991); en la actualidad continúa siendo objeto de estudio dada su relevancia para el desempeño deportivo (Mead, Drowatzky y Hardin-Crosby, 2000; Junge, Dvorak, Rösch, Graf-Baumann, Chomiak y Peterson, 2000; Andersen y Williams, 1999). Según Sanabria (1995), el efecto del ejercicio sobre el tiempo de reacción y en general sobre la condición mental es mayor al realizar ejercicio crónico de tipo aeróbico, que cuando se realiza ejercicio de tipo anaeróbico sobre el cual no se reportan efectos.

Otro de los procesos cognitivos que han recibido importante atención por parte de los científicos es la memoria, la cual consta de tres etapas: codificación (acústica, visual o semántica), almacenamiento (agrupamiento, organización) y recuperación. Desde este modelo, la información debe ser percibida y codificada, tras ser mantenida por un determinado intervalo de tiempo es almacenada, y finalmente puede ser recuperada para ser reproducida (Fernández, Moya e Iñiguez, 1999).

Diferentes autores y modelos teóricos identifican distintos tipos de memoria. El modelo de Atkins y Shiffrin (1971), el más conocido y dentro del que se enmarca gran parte de la investigación, distingue tres estructuras: la memoria sensorial (MS) que registra información durante un tiempo no mayor a un segundo, tiempo suficiente para que la información sea transferida a otra estructura y dentro de la que se identifican dos modalidades sensoriales, la memoria icónica y la ecóica; la memoria a corto plazo (MCP), que guarda información limitada por un periodo inferior a un minuto y desde la cual actúan los mecanismos de control; y la memoria a largo plazo (MLP) que es relativamente permanente e ilimitada (Puente, Moya y Mayor, 1998).

La duración del material que ingresa a la MCP determina el nivel de recuerdo, entre más tiempo permanezca en la memoria es mas probable que se establezcan relaciones con otros materiales almacenados en la MLP; tal duración depende de la significación del material y de la motivación principalmente. En cuanto a la capacidad de la MCP, se ha establecido la cantidad de siete elementos como el número promedio de elementos que normalmente se pueden almacenar en ésta (Puente, Moya y Mayor, 1998). Para Pearce (1998), el olvido en la MCP se debe a la falta de estímulo persistente, debido a que la MCP almacena la información por poco tiempo y se deshace de ella tan pronto como sea necesario retener otra.

La eficacia de la memoria según Hess y Blanchard (1999), es relativamente estable hasta los 40 años de edad y el declive que presenta a partir de entonces es generalmente precipitado. La capacidad de memoria, así como la mayoría de las facultades psicológicas y físicas, se van deteriorando como parte natural del proceso de envejecimiento; se produce un enlentecimiento y una menor eficiencia del funcionamiento cognitivo y esto hace que la respuesta a los estímulos sea más lenta. Durante este periodo se producen cambios sobre cada sistema de memoria; se parte del supuesto de que gran parte de las deficiencias que ocurren no tienen una base biológica por no estar asociados a enfermedades del sistema nervioso central, o si la tienen pueden ser rehabilitados, dada la enorme plasticidad del sistema nervioso (Fernández, Moya e Iñiguez, 1999). En relación con la actividad física, Sanabria (1995) encontró que el mayor beneficio sobre la memoria se daba como efecto crónico entre las personas de 46 a 60 años de edad, con ejercicio de tipo aeróbico.

Otra de las características asociadas con el proceso de envejecimiento es una disminución de la capacidad para hacer 
trabajo físico y mental (Meléndez, 2000). Un estudio realizado por Sallis (2000), con niños, adolescentes y adultos, reveló que conforme se envejece la tendencia a disminuir la actividad física es significativa, de manera que tanto el tipo de actividad como la intensidad disminuyen en las mismas proporciones; adicionalmente encontró que las mujeres tienden a la vida sedentaria más que los hombres (Evans, 1999; Sallis, 2000).

Instituciones de carácter internacional como el Colegio Americano de Medicina Deportiva -ACSM- (1999) recomienda para las poblaciones mayores de 50 años caminar o participar en programas de entrenamiento muscular. Dicha recomendación nace a partir de que aunque tradicionalmente solo se ha recomendado ejercicio de tipo aeróbico para incrementar el rendimiento cardiovascular, el entrenamiento con pesas se sugiere como un importante componente del programa de acondicionamiento físico de las personas mayores quienes experimentan una pérdida importante de la masa muscular (Evans, 1999 y Meléndez, 2000).

Dado que el trabajo investigativo realizado sobre el ejercicio y variables cognitivas ha estado principalmente centrado en los efectos a mediano y a largo plazo, es escaso el conocimiento acerca del ejercicio de corta duración y su efecto agudo en personas de la tercera edad, para quienes la fuerza es una de las cualidades físicas mas importantes para realizar sus tareas diarias autónomamente. Por lo tanto, el presente estudio tiene como propósito determinar si una sesión de ejercicio con pesas a distintas intensidades, produce cambios en la memoria a corto plazo y en el tiempo de reacción en adultas mayores.

\section{METODOLOGÍA}

\section{Sujetos}

Participaron voluntariamente 20 mujeres, con una edad promedio de $55 \pm 6$ años, 10 de ellas integrantes de un curso de manualidades (grupo sedentario); las otras 10 participantes de un programa de acondicionamiento físico en la Universidad de Costa Rica con al menos dos años de experiencia.

\section{Instrumentos}

Se aplicaron dos tests, el Verbal Script Digit Span $(r=0.79)$, para medir la memoria auditiva, del cual sólo se ejecutó la primera parte en la que el evaluador presenta verbalmente una secuencia de números que deben ser repetidos en el mismo orden por la persona evaluada. Consta de siete niveles, con 3 dígitos en el primero y uno adicional por nivel superior. Al fallar en dos intentos en un mismo nivel se da por terminada la prueba y se contabiliza el último nivel superado (Solera y Salazar, 2001). Para el estudio se elaboraron tres plantillas diferentes, para usar una por sesión y evitar así posible efecto de aprendizaje.

El segundo fue el test de la mano de Nelson $(r=0.58)$ para medir el tiempo de reacción. En éste, el sujeto coloca el antebrazo y la muñeca descansando sobre una mesa; entre el dedo pulgar e índice se coloca verticalmente una regla con marcas en unidades de tiempo. El sujeto debe asir lo más rápido posible la regla en el momento en que la vea caer. El puntaje obtenido será el que quede señalado bajo su dedo pulgar, el cual indica el tiempo tardado en reaccionar al estímulo (Solera y Salazar, 2001).

Para las sesiones experimentales se utilizaron seis máquinas del gimnasio de la Escuela de Educación Física de la Universidad de Costa Rica, en las que se realizaron los ejercicios durante las sesiones experimentales, así como las respectivas pruebas de 5RM: extensor de rodilla, flexor de rodilla, press de pierna, press articulado, halón de polea y remo con polea. 


\section{Procedimiento}

Inicialmente se presentó la información sobre las condiciones del estudio entre las integrantes de los dos tipos de programas (manualidades y acondicionamiento físico). Las interesadas en participar firmaron un formulario de consentimiento, quedando conformados los dos grupos: sedentario y entrenado.

Se realizó la prueba de 5RM ( $r=$ 0.77-0.90 para el tren superior y $r=0.60$ 0.80 para el tren inferior, según Knutzen, Brilla y Caine, 1999) que equivale al 90\% de la fuerza máxima, con cada sujeto y para cada uno de los seis ejercicios que integraron las sesiones de ejercicios; a partir de estos valores se determinaron las cargas de trabajo correspondientes al 50\% y al $80 \%$ de ese 5RM. Las tres condiciones de tratamiento fueron aleatorizadas para cada sujeto. Cada sesión tuvo una duración de 45 a 50 minutos y el tiempo entre una y otra fue de una semana, manteniendo constante la hora de inicio para cada sujeto.
Previo al inicio del programa de ejercicios, las participantes asistieron a una sesión en la que se familiarizaron con las máquinas y aprendieron su uso. Cada sesión iniciaba con 10 minutos de calentamiento en bicicleta estática al $60 \%$ de la $\mathrm{FC}_{\text {máx.; }}$; posteriormente se aplicaban los tests, primero el de memoria y enseguida el de Nelson, mediciones que se repetían en el mismo orden inmediatamente se terminaba la sesión de ejercicio.

El protocolo para cada condición fue el siguiente:

1. $50 \%$ de fuerza máxima: tres series de 15 repeticiones, con 30 segundos para recuperación después de cada serie; incluyó seis ejercicios diferentes: extensión de rodilla, flexión de rodilla, press de pierna, press articulado, halón de polea de frente y remo con polea.

2. $80 \%$ de fuerza máxima: cuatro series de ocho repeticiones, con los mismos ejercicios.

3. Control: las sujetos permanecían en reposo, sentadas, durante el mismo tiempo y en las mismas condiciones ambientales.

Tabla 1. Estadística descriptiva $(M \pm D S)$ y tamaño del efecto (TE) en memoria auditiva y tiempo de reacción

\begin{tabular}{|c|c|c|c|c|c|c|}
\hline \multirow{2}{*}{ Grupo } & \multicolumn{2}{|c|}{ Memoria (niveles) } & \multirow[b]{2}{*}{ TE } & \multicolumn{2}{|c|}{ Tiempo de Reacción (s) } & \multirow[b]{2}{*}{ TE } \\
\hline & Pre-test & Post-test & & Pre-test & Post-test & \\
\hline \multicolumn{7}{|l|}{ Entrenadas } \\
\hline $50 \%$ & $4.8 \pm 1.81$ & $5.2 \pm 1.62$ & 0.22 & $0.174 \pm 2.748$ & $0.164 \pm 3.103$ & 0.03 \\
\hline $80 \%$ & $4.5 \pm 1.27$ & $4.7 \pm 1.34$ & 0.16 & $0.170 \pm 2.332$ & $0.164 \pm 2.402$ & 0.02 \\
\hline Control & $4.3 \pm 1.70$ & $5.1 \pm 1.97$ & 0.47 & $0.177 \pm 2.811$ & $0.167 \pm 2.705$ & 0.03 \\
\hline \multicolumn{7}{|l|}{ Sedentarias } \\
\hline $50 \%$ & $4.0 \pm 0.94$ & $4.4 \pm 1.07$ & 0.43 & $0.174 \pm 1.354$ & $0.160 \pm 2.085$ & 0.10 \\
\hline $80 \%$ & $4.1 \pm 0.74$ & $4.2 \pm 0.79$ & 0.14 & $0.180 \pm 2.004$ & $0.159 \pm 2.347$ & 0.11 \\
\hline Control & $4.1 \pm 0.74$ & $4.3 \pm 0.95$ & 0.27 & $0.176 \pm 1.687$ & $0.176 \pm 2.648$ & 0.00 \\
\hline
\end{tabular}

\section{Análisis de datos}

Después del análisis descriptivo de las variables en términos de promedios y desviaciones estándar, se determinó la varianza explicada con el Eta al cuadrado $\left(\eta^{2}\right)$ y se realizó un ANOVA mixto de 3 vías de $2 \times 3 \times 2$ (grupos $\times$ condiciones $\times$ mediciones), para cada una de las dos variables dependientes.

\section{RESULTADOS}

A continuación se presenta la estadística descriptiva correspondiente a los dos grupos, en las dos mediciones para cada condición, en las dos variables dependientes 
(Tabla 1). Los resultados de los análisis de varianza realizados para cada una de las variables medidas y el índice de varianza explicada, se presentan en la Tabla 2.

Tabla 2. ANOVA y varianza explicada para cada variable

\begin{tabular}{llccc}
\hline Variable & \multicolumn{1}{c}{ Fuente } & $\boldsymbol{F}$ & $\boldsymbol{p}$ & $\boldsymbol{\eta}^{\mathbf{2}} \mathbf{( \% )}$ \\
\hline Memoria & Grupo & 1.719 & 0.206 & 8.7 \\
& Condición & 0.547 & 0.584 & 2.9 \\
& Medición & $7.025^{*}$ & 0.016 & 28.1 \\
& Grupo x condición & 0.373 & 0.691 & 2.0 \\
& Grupo x medición & 0.781 & 0.389 & 4.2 \\
& Condición x medición & 0.846 & 0.438 & 4.5 \\
& Grupo x condición x medición & 0.672 & 0.517 & 3.6 \\
\hline \multirow{3}{*}{ Tiempo de reacción } & Grupo & & & \\
& Condición & 0.250 & 0.876 & 0.1 \\
& Medición & 0.713 & 0.497 & 3.8 \\
& Grupo x condición & $28.933^{*}$ & 0.000 & 61.6 \\
& Grupo x medición & 0.244 & 0.785 & 1.3 \\
& Condición x medición & 0.300 & 0.590 & 1.6 \\
& Grupo x condición x medición & 1.645 & 0.207 & 8.4 \\
& & 3.034 & 0.061 & 14.4 \\
\hline
\end{tabular}

$* p<.05$

No se halló interacción significativa entre los tres factores, ni entre pares de factores para ninguna de las variables dependientes. Sin embargo, el nivel inicial de memoria tiende a ser levemente superior entre las mujeres activas físicamente con respecto a las sedentarias; así mismo se observa en el grupo de sedentarias que en la condición control no hubo mejoría en el post-test como sí la hubo en las dos condiciones de ejercicio, aunque tales diferencias no fueron estadísticamente significativas (Figura 1).

Con respecto a los efectos principales, se hallaron diferencias significativas entre las mediciones, tanto para memoria auditiva como para tiempo de reacción, independientemente de la condición de tratamiento y del grupo experimental, lo que significa que el promedio general en la medición del pos-test fué significativamente superior que en el pre-test, en las dos variables. Así, tanto las sedentarias como las entrenadas, y las que hicieron ejercicio a diferentes intensidades como las que no lo hicieron, mejoraron en el post-test (Figuras 2 y 3 ).

\section{DISCUSIÓN}

No se encontró apoyo a la hipótesis de investigación según la cual se esperaba encontrar un incremento significativo en las variables de memoria y tiempo de reacción como respuesta al ejercicio con pesas en adultas mayores.

Son muchos los estudios sobre los que se apoya la conclusión de que el ejercicio crónico, tanto aeróbico como anaeróbico, favorece el desempeño cognitivo del ser humano, aunque el tamaño de tal efecto es dependiente de varios factores, unos relacionados con las características del ejercicio (tipo, frecuencia e intensidad) y otros relacionados con las características de los sujetos (edad, sexo y nivel de entrenamiento principalmente) (Emery $\mathrm{y}$ Blumenthal, 1991). En tal sentido, Sanabria (1995) reporta que los mayores beneficios del ejercicio sobre la memoria se presentan 
en las personas con edades comprendidas entre 46 y 60 años.

Sin embargo, en cuanto al efecto agudo los resultados son menos concluyentes además por ser más escasas las investigaciones. En lo que respecta a memoria, Sanabria (1995) señala que se ha encontrado efecto agudo significativo después de sesiones de 80 a 90 minutos de ejercicio aeróbico, mientras que el tiempo de reacción disminuye con sesiones de 20 a 60 minutos de duración. Las sesiones experimentales del presente estudio tuvieron en promedio una duración de 50 minutos; quizá al incrementar la duración de la sesión los resultados podrían ser diferentes por lo que se sugiere estudiar sesiones de ejercicio anaeróbico, de diferente duración. Según el meta-análisis de Sanabria, se encontró un efecto positivo y significativo del ejercicio aeróbico sobre la memoria $(\mathrm{TE}=1.07, p<$ .001 ); los pocos estudios que examinaron el ejercicio anaeróbico, solo lograron efectos positivos sobre el tiempo de reacción.

Figura 1. Promedios de tiempo de reacción en función de grupo, condición y medición

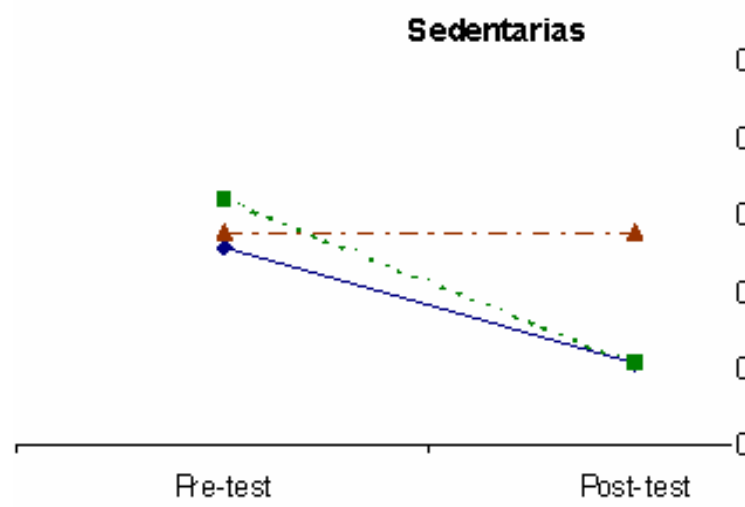

En la presente investigación, los resultados de tiempo de reacción mostraron una tendencia diferente entre las condiciones de ejercicio y la de control especialmente en el grupo de mujeres sedentarias, aunque la interacción correspondiente no alcanzó el nivel de significancia a pesar de explicar el $14.4 \%$ de la varianza total de la variable. Con respecto a la variable memoria a corto plazo se observaron niveles iniciales más altos en las mujeres activas físicamente que en las sedentarias, aunque igualmente tales diferencias tampoco fueron significativas.

Podría ser que el tamaño de la muestra fue muy pequeño y por consiguiente afectó la potencia al diseño, lo cual señala la posibilidad de hallar un efecto agudo importante si se trabaja con una muestra
Entrenactas

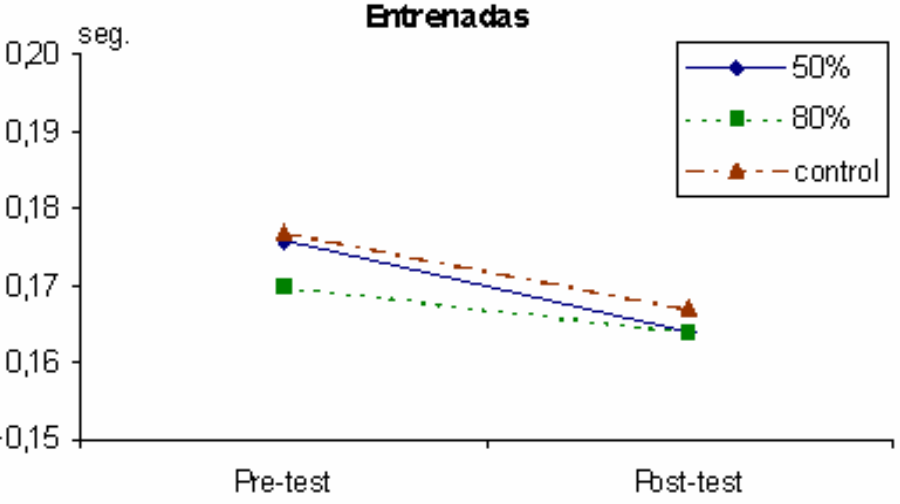

mayor. Además del tamaño de la muestra, pudo presentarse un efecto de aprendizaje que encubrió el efecto del ejercicio, dado por la familiarización tanto con el test (a pesar de haber utilizado en el test de memoria secuencias numéricas diferentes para cada medición) como con la respuesta que del sujeto se demanda. Este se deduce del cambio en el post-test observado en el grupo control y cuyo patrón no fué significativamente diferente con respecto al observado en los dos grupos experimentales. Sin embargo, Alfaro y Salazar (2001) no hallaron efecto de aprendizaje en la condición control de su estudio, en ninguno de los grupos etáreos que analizaron y habiendo utilizado repetidamente el mismo test para medir memoria. 
Figura 2. Promedios generales de pre-test y posttest en memoria auditiva

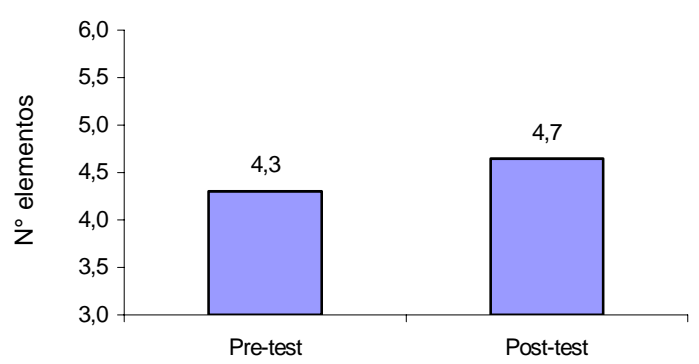

Según Emery y Blumenthal (1991) sólo Molloy y cols (1988) habrían examinado el efecto agudo del ejercicio sobre el funcionamiento neuro-psicológico en adultos mayores, encontrando una mejora en memoria lógica y en el estado mental general. Sin embargo se cuestionan tales resultados por debilidades metodológicas del diseño y del análisis estadístico.

Varios de los estudios que han reportado cambios sobre las variables cognitivas producidos por el ejercicio, los explican en función del favorecimiento del transporte y la utilización de oxígeno en el cerebro, el aumento en el metabolismo de la glucosa a nivel celular que a su vez permite una adecuada provisión y utilización de la energía sin disminuir los niveles de glucosa en el sistema nervioso central, y a una mejor renovación de, neurotransmisores (Sanabria, 1995; Morel, 1996; Aguirre, 1999; y Alfaro y Salazar, 2001). Sin embargo, los cambios observados bajo la condición control no pueden ser explicados por tal mecanismo fisiológico.

Emery y Blumenthal (1991) sostienen que no están claros los mecanismos por los cuales el ejercicio afecta el funcionamiento cognitivo y sugieren que estos pueden variar a través de grupos e individuos. Sugieren que los modelos que expliquen los efectos del ejercicio deben considerar tales variaciones en la investigación futura. Igualmente señalan que es importante comprender la diferencia entre el efecto crónico y el agudo para ayudar a
Figura 3. Promedios generales de pre- test y post- test en tiempo de reacción

seg.

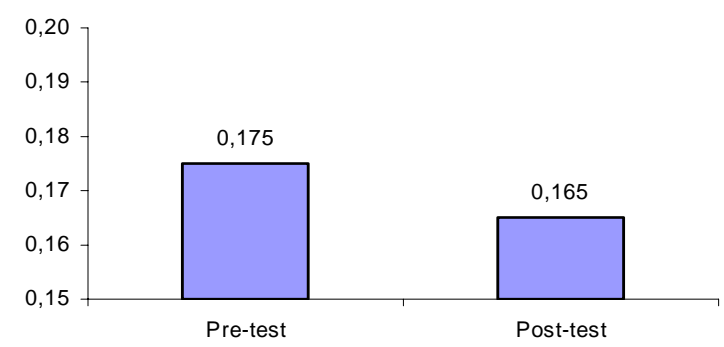

esclarecer los mecanismos por los cuales el ejercicio influye sobre el funcionamiento cognitivo.

Igualmente, Dishman (2000) afirma que la evidencia a favor de las hipótesis fisiológicas para explicar los mecanismos mediante los cuales el ejercicio provoca beneficios psicológicos, no es concluyente y sugiere que para llegar a una plena comprensión de estos, es necesario hacer uso de los conocimientos y técnicas que las neurociencias ofrecen para el estudio de los efectos del ejercicio.

De otra parte, Berger y Molt (2000) concluyen a partir de una revisión de literatura, que los efectos del ejercicio sobre los estados de ánimo no difieren del efecto que producen otras técnicas como la relajación, la lectura, el tai-chi e incluso la interacción social, lo cual podría explicarse desde la hipótesis de la distracción (Etnier y cols., 1997). Hasta el momento, no se conocen estudios sobre ejercicio y función cognitiva que hayan intentado poner a prueba tal hipótesis la cual fue originalmente estructurada en el marco de los procesos emocionales (Morgan, 1985).

Es posible que el incremento en los procesos cognitivos observados al finalizar la sesión experimental, tanto en las dos condiciones de ejercicio como en la que permaneció en reposo e interactuando, pudiera explicarse a partir del mecanismo de la distracción, según el cual el efecto psicológico no es causado por el ejercicio en sí mismo, sino por el cambio temporal que 
hace el sujeto al pasar de un ambiente estresante a una situación diferente que distrae su atención (Morgan, 1985). Sin embargo, esta hipótesis amerita ser sometida a comprobación científica, dadas las implicaciones que para efectos prácticos podría conllevar en cuanto a implementación de programas integrales de promoción de salud.

\section{REFERENCIAS}

Aguirre, L. (1999). Efectos crónicos del entrenamiento de fuerza sobre procesos cognitivos en personas mayores de 60 años. Tesis de licenciatura no publicada, Universidad Nacional. San José.

Alfaro, Y. y Salazar, W.(2001). Efecto agudo del ejercicio físico en la inteligencia y la memoria en hombres, según la edad. Revista de Ciencias del Ejercicio y la Salud, 1(2), 1-11.

Andersen, M.B. y Williams, J.M. (1999) Athletic injury, psychosocial factors and perceptual changes during stress. Journal of Sport Sciences, 17(9), 735-41

Atkinson, R.C. y Shifrin, R.M. (1971). The control of short-term memory. Scientific American, 225, 82-90.

Berger, B. y Motl, R. (2000). Exercise and mood: a selective review and synthesis of research employing the poms. Journal of Applied Sport Psychology, 12 (1), 69-92.

Bly, M.B. y Rumelhart, D.E. (1999). Cognitive science. CA: Academic Press.

Clark, T.J. (1996). The relationship between physical self- perceptions and functional muscular strength in young adult females [CD-ROM]. Microform Publications, International Institute for Sport \& Human Performance, University of Oregon. Resumen de SilverPlatter Archivo: SPORT Discuss. Identificador: 410962.

Dishman, R.K. y Jackson, E.M. (2000). Exercise, fitness and stress. International Journal of Sport Psychology, 31 (2), 175-203.

Emery, Ch. y Blumenthal, J. (1991). Effects of physical exercise on psychological and cognitive functioning of older adults. Exercise and Aging, 13(3), 99-105.

Etnier, J., Salazar, W., Landers, D., Petruzzello, S., Han, M. y Novell, P. (1997). The influence of physical fitness and exercise upon cognitive functioning: A meta-analysis. Journal of Sport \& Exercise Psychology, 19, 249-277.

Evans, W. (1999). Exercise training guidelines for elderly. Medicine and Science in sports and Exercise, 31(1), 12-17.
Fernández, B. R., Moya, F. R. e Iñiguez, M. J. (1999). ¿Qué es la psicología de la vejez? Madrid: Biblioteca Nueva.

García, J., Navarro, M., Ruiz, J. (1996). Bases teóricas del entrenamiento deportivo: principios $y$ aplicaciones. Madrid: Gymnos.

Hess, T. y Blanchard, F. (1999). Social cognition and aging. (Eds.). California: Academic Press.

Hughes, C.A. (1973). Comparison of the effects of four teaching techniques of body conditioning upon physical fitness and self-concept. [CDROM]. Tesis Doctoral, Institute for Sport and Human Performance, University of Oregon. Resumen de SilverPlatter Archivo: SPORT Discuss. Identificador: 16433.

Junge, A., Dvorak, J., Rösch, D., Graf-Baumann, T., Chomiak, J. y Peterson, L. (2000). Psychological and sport-specific characteristics of football American Journal of Sports Medicine, 28(5Suppl), 22-8.

Knutzen, K., Brilla, L y Caine, D. (1999). Vality of 1RM prediction for older adults. Journal of Strength and Conditioning Research, 13 (3),242246.

Lox, C., Burns, S., Treasure, D. y Wasley, D. (1999). Physical and psychological predictors of exercise dosage in healthy adults. Medicine and Science in Sports and Exercise, 31 (7), 1060-1064.

Márquez, M. y Campos, C. (2001). Efecto de un programa de ejercicios con pesas en el porcentaje de grasa y la fuerza en mujeres mayores. Revista de Ciencias del Ejercicio y la Salud, 1 (2), 31-37.

Maxwell, K. (1994). The effects of weight training program on emotional web-being and body image of college females. [CD-ROM]. Microform Publications, International Institute for Sport \& Human Performance, University of Oregon. Resumen de SilverPlatter Archivo: SPORT Discuss. Identificador: 367476.

Mead, T. P., Drowatzky, J.N. y Hardin-Crosby, L. (2000). Positive and negative stimuli in relation to tennis players' reaction time. Perceptual and Motor Skills, 90(1), 236-40.

Meléndez, A. (2000). Actividades fisicas para mayores: las razones para hacer ejercicio. Madrid: Paidotribo.

Moreira, A. R. (1993). Efectos crónicos y agudos del ejercicio en los procesos cognitivos. Tesis de Licenciatura no publicada, Universidad de Costa Rica. San José.

Morel, V. (1996). Relaciones entre la funcionalidad, el nivel de actividad física, el estado cognitivo y anímico de adultos mayores participando en programas recreativos de actividad física. Memorias III Simposio Internacional de Ciencias del Deporte y la Salud. San José: Universidad de Costa Rica.

Morgan, W.P. (1985), Select psychological factors limiting performance: A mental health model. En: D.H Clark y H.M. Eckert (Eds.). Limits of 
human performance (pp. 70-80). Champaign, IL: Human Kinetics.

Pearce, J. (1998). Aprendizaje y cognición. Barcelona: Editorial Ariel, S.A..

Perrig-Chiello, P., Perrig, W.J., Ehrsam, R.P., Staehelin, H. y Krings, F. (1998). The effects of resistance training on well-being and memory in elderly volunteers. Age and Ageing 27, 469-475.

Pollock, M. y Evans, W. (1998). Resistance training for health and disease: introduction. Medicine and Science in Sports and Exercise, 31 (1), 1011.

Puente, A.., Moya, S. y Mayor, M. (1998). Cognición $y$ aprendizaje: fundamentos psicológicos. Madrid: Ediciones Pirámide.

Riera J. y Cruz, J. (1991) Psicología del deporte: aplicaciones y perspectivas. Barcelona, Martínez Roca.
Roca J. (1997). Tiempo de reacción en el deporte. En J. Cruz (Ed.) Psicología del deporte (pp. 43-74). Madrid: Editorial Síntesis.

Sallis, J.F. (2000). Age- related decline in physical activity: a synthesis of human and animal studies. Medicine and Science in Sports and Exercise, 32 (9), 1598-1600.

Sanabria, I. (1995). Meta-análisis sobre los efectos del ejercicio en parámetros cognitivos. Tesis de Licenciatura no publicada, Universidad de Costa Rica. San José.

Solera, A. y Salazar, W. (2001). Efectos de la deshidratación y la rehidratación sobre los procesos cognitivos de tiempo de reacción, memoria auditiva y percepción visual. Revista de Ciencias del Ejercicio y la Salud, 1(1), 1-9.

Valliant, P. y Asu, M. (1984). Exercise and its effects on cognition and physiology in older adults. Perceptual and Motor Skills, 61(3P), 1031-1038. 